\title{
Neurotechnologien aus der Perspektive einer Theorie konkreter Subjektivität
}

Zusammenfassung: Die Manipulation neuronaler Strukturen durch Neurotechnologien geht gerade bei Anwendungen am Gehirn oftmals mit intendierten oder unerwünschten Einflüssen auf geistig-psychische Aspekte menschlicher Lebensvollzüge einher. Daher sind für ein fundiertes Verständnis neurotechnologischer Entwicklungen und Anwendungen anthropologische Überlegungen über das Verhältnis des Bewusstseins zu den technisch manipulierbaren organismischen Strukturen bzw. über die psycho-physische Konstitution des Menschen unumgänglich. Vor diesem Hintergrund verfolgt der vorliegende Beitrag das Ziel, durch die Skizze einer Theorie konkreter Subjektivität einen allgemeinen anthropologischen Reflexionsrahmen für das Feld der Neurotechnologie zu entwickeln, der einerseits den spezifischen irreduziblen Eigenschaften des menschlichen Bewusstseins und andererseits der realen Möglichkeit seiner Beeinflussung durch Neurotechnologien Rechnung tragen kann. Hierfür wird zunächst die irreduzible Grundstruktur des Bewusstseins aufgezeigt. Indem sie dabei zugleich als unhintergehbare Voraussetzung für das Erfüllen von Rationalitätsstandards lebensweltlicher und wissenschaftlicher Praktiken ausgewiesen wird, können die gerade auch in den Neurowissenschaften - weit verbreiteten reduktiv-naturalistischen Deutungen des Bewusstseins, die seine vollständige Reduzierbarkeit auf physische kausal-funktionalen Strukturen behaupten, als unzulänglich zurückgewiesen werden. Den Kern der Theorie konkreter Subjektivität bildet die anschließende Bestimmung des Menschen als binnendifferenzierte Einheit, in der Bewusstsein und Organismus als zwei zu unterscheidende, nicht aufeinander reduzierbare und einander bedingende Momente verschränkt sind. Da somit einerseits die spezifischen irreduziblen Charakteristika des Bewusstseins, andererseits seine konstitutive Einbettung in organismische Strukturen und damit auch in einen technisch manipulierbaren Naturzusammenhang berücksichtigt werden, erweist sich diese anthropologische Perspektive als geeigneter Reflexionsrahmen, um das allgemeine Verständnis der neurotechnologischen Einflussnahme auf menschliche Lebensvollzüge mitsamt ihren geistig-psychischen Aspekten schärfen sowie konkrete Anwendungsfälle differenziert beurteilen zu können - beides wird in einem abschließenden Teil exemplarisch demonstriert. 


\section{Das Phänomen der Neurotechnologie - anthropologischer Reflexionsbedarf}

Die enormen Fortschritte moderner Neurowissenschaften in der Ergründung der Struktur und Funktion des menschlichen Gehirns ebneten den Weg für das sich dynamisch entwickelnde Feld der Neurotechnologie. ${ }^{1}$ Damit steht eine Entwicklung im Raum, die einen fundamentaleren Zugriff auf den Menschen als jede andere technische Entwicklung zuvor zu ermöglichen scheint. Denn bei der technischen Manipulation neuronaler Strukturen (vor allem des Gehirns) geht es nicht mehr wie in anderen biotechnischen Kontexten vorrangig um die Beeinflussung rein physiologischer Prozesse. Sondern insofern das Gehirn „der zentrale Ort bewusstseinsrelevanter physiologischer Prozesse“ (Fuchs 2013, 68) ist, geht es um die Beeinflussung des Menschen in seinem personalen Dasein als psychisch-geistiges, sich seiner selbst bewusstes, selbstbestimmtes und handelndes Subjekt.

Solche Einflüsse auf mental-psychische Aspekte zeigen sich zum einen als ungewollte Nebenwirkungen im Rahmen etablierter neurotechnologischer Therapieansätze in der Medizin. Beispielsweise können beim Einsatz der Tiefen Hirnstimulation ${ }^{2}$ zur Behandlung motorischer Störungen gravierende Auswirkungen auf kognitive Fähigkeiten oder das affektive Erleben der Patienten auftreten (vgl. z. B. Gharabaghi/Freudenstein/Tatagiba 2005, 65f.). Zum anderen gibt es neurotechnologische Anwendungen und Entwicklungsziele, bei denen ein bestimmter Einfluss auf die Psyche bzw. das Bewusstsein gerade intendiert wird. Dabei weisen solche anvisierten - teils schon realisierten, teils noch visionären Manipulationen mental-psychischer Aspekte ein weites Spektrum auf, das sowohl therapeutisch-wiederherstellende als auch bestimmte Funktionen verbessernde bzw. erweiternde Eingriffe umfasst: von neuen Therapieansätzen psychischer Erkrankungen (z. B. therapierefraktärer Depressionen) durch das Verfahren Tiefer Hirnstimulation (vgl. z. B. Holtzheimer/Mayberg 2011; Mi 2016) über die ange-

1 Unter dem Begriff Neurotechnologie seien im Folgenden alle technischen Mittel und Methoden verstanden, die einen direkten Kontakt zwischen einem technischen Element und dem Nervensystem ermöglichen. Der Kontakt basiert dabei auf einer elektrisch stimulierenden oder bioelektrische Signale decodierenden Elektrode. In ,integrierten Systemen' werden beide Verfahren kombiniert (siehe für einen Überblick z. B. Clausen 2008, 40 - 45; Müller/Rotter 2017).

2 Bei der Tiefen Hirnstimulation werden je nach Indikation verschiedene subkortikale, ,tiefe Hirnstrukturen durch eine implantierte Elektrode stimuliert. Siehe für eine Auseinandersetzung mit dieser Methode auch Olivia Mitscherlich-Schönherrs Beitrag zum vorliegenden Band. 
strebte Wiederherstellung und Verbesserung kognitiver Fähigkeiten ${ }^{3}$ bis hin zu Visionen, die von einer so großen technischen Kontrollierbarkeit des Bewusstseins ausgehen, dass etwa Erinnerungen auf einen implantierten ,GedächtnisChip' gespeichert oder das ganze Bewusstsein auf einen Computer geladen werden könnte (vgl. z. B. Hansmann 2018, 46 f.; Hildt 2005, 134).

In Anbetracht solcher Zugriffe auf zentrale Aspekte unseres personalen Daseins sind anthropologische Überlegungen für das Feld der Neurotechnologie von besonderer Relevanz. Die Deutung der Einflussnahme neurotechnologischer Anwendungen auf mental-psychische Aspekte, ihre ethische Beurteilung, die Einschätzung ihrer Grenzen und Möglichkeiten sowie die davon abhängige Ausrichtung der neurowissenschaftlichen Forschung hängen maßgeblich von anthropologischen Vorannahmen darüber ab, wie man Bewusstsein in seiner $\mathrm{Ab}$ hängigkeit von neuronalen Prozessen bzw. die psycho-physische Konstitution des Menschen begreift. Anders gesagt steht im Kontext der Neurotechnologien hinter jeder ethischen Reflexion, hinter jedem neuen Entwicklungsziel und hinter jedem therapeutischen oder verbessernden „Zugriff auf das Gehirn [...] jeweils schon ein bestimmtes Menschenbild“ (Clausen/Müller/Maio 2008, 8), das zur adäquaten Beurteilung eines Eingriffs oder eines anvisierten Ziels expliziert werden muss.

Dabei lässt sich beobachten, dass solchen Beurteilungen und Überlegungen im Kontext der Neurowissenschaften und Neurotechnologie häufig ein reduktivnaturalistisches Menschenbild zugrunde liegt, gemäß dem sich alle Aspekte des Menschseins, auch die mental-psychischen (Emotionen, kognitive Fähigkeiten etc.), auf materielle physiologische bzw. neuronale Abläufe zurückführen lassen, also ein Subjekt letztlich nicht mehr als und identisch mit seinem Gehirn ist:

„You are your brain. The neurons interconnecting in its vast network, discharging in certain patterns modulated by certain chemicals, controlled by thousand feedback networks - that is you. And in order to be you, all of those systems have to work properly.“ (Gazzaniga 2005, 31)

Somit werden etwa psychische Krankheiten als reine Störungen des Gehirns verstanden, die man folglich im Zuge des wissenschaftlichen Fortschritts allein durch medizinisch-technische Manipulationen neuronaler Strukturen heilen könne (vgl. z. B. Elger et al. 2004, 36).

Solche reduktionistischen anthropologischen Vorannahmen im Feld der Neurotechnologie basieren auf der weit verbreiteten - sicherlich durch den Erfolg der modernen Naturwissenschaften motivierten - ontologischen Grundhaltung,

3 So gibt es beispielsweise Versuche, einen ,Hippocampus-Chip، für die Wiederherstellung des Langzeitgedächtnisses bei hippocampalen Degenrationen zu entwickeln (vgl. Berger et al. 2005). 
dass alles Wesentliche der Wirklichkeit naturwissenschaftlich erfasst werden könne (vgl. z. B. Beckermann 2012, 6) und sich somit in physischen wirkkausalfunktionalen Strukturen erschöpfe. Alles, was sich hingegen dem methodisch auf kausal-funktionale Kategorien beschränkten naturwissenschaftlichen Weltzugang entzieht - etwa ein Gedanke oder ein Sinneseindruck -, wird als ontologisch sekundäres und kausal irrelevantes Phänomen angesehen, das von der ontologisch fundamentalen Ebene der naturwissenschaftlich fassbaren (also kausalfunktionalen) Naturzusammenhänge vollständig bestimmt und insofern auf diese zurückführbar sei. ${ }^{4}$ Wirklichkeit wird demnach als rein wirkkausal-funktionales „Geschehen bzw. die Folge eines bloßen Geschehens“ (Cramm 2008, 44) verstanden, wobei den fundamentalen physischen Strukturen exklusive Kausalkräfte zukommen, die bottom-up alle anderen Phänomene - also auch mental-psychische Aspekte des Menschen - vollständig bestimmen (vgl. Müller 2018, 91).

Diese reduktiv-naturalistische Auffassung der Wirklichkeit und des Menschen sieht sich jedoch mit erheblichen Schwierigkeiten konfrontiert: Zum einen bedeutet sie einen fundamentalen Bruch mit unserem lebensweltlichen Selbstverständnis, nicht ausschließlich durch physische Abläufe determinierte materielle Konglomerate, sondern wesentlich auch psychisch verfasste Subjekte zu sein, die sich denkend und prinzipiell frei an Zwecken und Gründen orientieren können (vgl. Müller 2015, 31-33). Zum anderen wird, wie wir noch sehen werden, eine naturalistische Reduktion des Bewusstseins auf ein ,blind‘ ablaufendes Naturgeschehen den Rationalitätsstandards lebensweltlicher und wissenschaftlicher Praktiken nicht gerecht, also auch nicht der auf rational-wissenschaftlichen Erkenntnissen aufbauenden Entwicklung und Anwendung von Neurotechnologien. ${ }^{5}$

Angesichts dieser Probleme reduktiv-naturalistischer Deutungen möchte ich im Folgenden einen anthropologisch adäquateren Reflexionsrahmen für das Feld der Neurotechnologie vorstellen, der nicht nur der realen Möglichkeit neurotechnologischer Einflussnahme auf den Menschen und sein Bewusstsein, sondern auch unserem lebensweltlichen Selbstverständnis und einem zu rationalen Praktiken befähigendem Denkvermögen - der Voraussetzung erfolgreicher neurotechnologischer Entwicklungen - Rechnung tragen kann. Hierfür wird in einem ersten Schritt eine Theorie konkreter Subjektivität skizziert, die den Menschen als psycho-physische Einheit begreift, in der Bewusstsein und Organismus als zwei konstitutive, einander bedingende und nicht aufeinander reduzierbare Momente

4 Siehe zum Zusammenhang von erfolgreicher naturwissenschaftlicher Wirklichkeitsbeschreibung und ontologischen reduktiv-naturalistischen Positionen auch Hoffmann 2013, 28-30 und Müller 2015, 32.

5 Darauf werde ich am Ende des Abschnitts 2.1 näher eingehen. 
verschränkt sind. Anschließend wird in einem zweiten Schritt exemplarisch demonstriert, dass sich eine solche Theorie konkreter Subjektivität eignet, um neurotechnologische Anwendungen und Entwicklungen aus anthropologischer Perspektive zu beleuchten, sei es, um das allgemeine Verständnis ihrer Grenzen und Möglichkeiten zu schärfen, sei es, um Deutungen konkreter Anwendungsfälle differenziert beurteilen zu können.

\title{
2 Skizze einer Theorie konkreter Subjektivität
}

Die vorliegende Skizze einer Theorie konkreter Subjektivität basiert vorrangig auf Überlegungen der Philosophen Wolfgang Cramer und Thomas Fuchs. Während mit Cramers transzendentalphilosophischem Ansatz die irreduzible Grundstruktur des Bewusstseins und seine gleichzeitig konstitutive Einbettung in einen Organismus aufgewiesen werden kann, liefert Fuchs, der phänomenologische und empirische Aspekte stärker berücksichtigt, das geeignete Instrumentarium, um die mit Cramer begründeten Bedingungsverhältnisse von Bewusstsein, Organismus und Natur zu konkretisieren.

\subsection{Die irreduzible Grundstruktur des Bewusstseins: Phänomenales Erleben und Denken als genuin aus und rückbezüglich für sich produzierende Aktivität eines Subjekts}

\begin{abstract}
„But no matter how the form may vary, the fact that an organism has conscious experience $a t$ all means, basically, that there is something it is like to be that organism. There may be further implications about the form of the experience; there may even (though I doubt it) be implications about the behavior of the organism. But fundamentally an organism has conscious mental states if and only if there is something that it is like to be that organism something it is like for the organism.“ (Nagel 1974, 436)
\end{abstract}

Thomas Nagel weist hier in seinem berühmten Aufsatz „What Is It Like to Be a Bat?" auf zwei konstitutive Aspekte eines jeden erlebten Bewusstseinszustandes hin: Der erste ist die phänomenale Qualität, die sich darin zeigt, dass es immer irgendwie ist bzw. sich immer irgendwie anfühlt, in einem Bewusstseinszustand zu sein, sei es einen Farbeindruck, eine leibliche Empfindung, einen abstrakten Gedanken oder alles gleichzeitig zu haben. Der zweite Aspekt geht notwendig mit dem ersten einher und ist das strukturelle Moment, dass ein Bewusstseinszustand und seine phänomenalen Qualitäten immer für ein konkretes Lebewesen sind, 
das diesen hat und dabei qualitativ erlebt. ${ }^{6}$ Das heißt zugleich, dass erlebte Bewusstseinszustände fundamental privat, also nur für das Subjekt sind, das sie erlebt, und sich nicht in eine öffentliche Dritte-Person-Perspektive übersetzten lassen (vgl. Brüntrup 2012, 13f.).

Dieser Für-es-Sein-Aspekt bedeutet nichts anderes, als dass es phänomenale Erlebensqualitäten (z.B. Farbeindrücke) nur insofern gibt, als ein Subjekt existiert, das sie erlebt. Anders gesagt existieren sie nicht als dem erlebenden Subjekt transzendente und von ihm unabhängige Eigenschaften des Naturzusammenhangs, auf die es sich im Sinne eines naiven Realismus einfach hinbeziehen könnte, sondern sie kommen nur innerhalb des Erlebens eines sie erlebenden Subjekts vor. Ein Baum mag dem Erleben transzendent sein, aber nicht der Farbeindruck seiner grünen Blätter. Da somit das Verhältnis von Erleben und erlebten Gehalten nicht so verstanden werden kann, dass Letztere schon außerhalb des Erlebens eines Subjekts existieren oder entstehen und sekundär auf welche Weise auch immer in dessen Erleben hineinkommen, muss das erlebende Subjekt zugleich der Ursprung der von ihm erlebten Gehalte sein (vgl. Cramer 1999, 28; 2012, 116). Erleben ist folglich als rückbezüglicher Zusammenhang zu verstehen, in dem das erlebende Subjekte die Gehalte, die es erlebt, genuin aus und aktiv für sich erzeugt (vgl. ders. 2012, 91-95).

Da sich diese rückbezüglich produzierende Aktivität kategorial von einem kausal-funktionalen Naturgeschehen unterscheidet, erweist sich Erleben als eigener, irreduzibler Modus von Wirklichkeit (vgl. ders. 1999, 32f.). Das heißt im Umkehrschluss, dass die oben skizzierte reduktiv-naturalistische Perspektive den spezifischen Eigenschaften des Erlebens nicht gerecht werden kann: Versucht man, Erleben auf die Ebene kausal-funktionaler Naturabläufe zurückzuführen und allein durch das Zusammenspiel physischer Kausaldispositionen zu erklären, so lassen sich weder der qualitative Charakter und die Privatheit des Erlebens noch die grundlegende rückbezügliche Struktur einholen, dass Erlebensgehalte für ein sie erlebendes Subjekt sind.

Nicht nur anhand des phänomenalen Erlebens, sondern auch anhand des Denkens, des Bewusstseinsmodus des begrifflichen Bestimmens, kann begründet werden, dass sich Bewusstsein als genuin aus und für sich produzierende Aktivität eines Subjekts charakterisieren lässt, die sich einer naturalistischen Reduktion auf wirkkausal-funktionale (neuronale) Strukturen entzieht. Denn jedes rationale Urteilen, Erkennen und Handeln setzt ein denkendes Subjekt voraus, das als genuiner Ursprung seiner Gedanken deren Inhalt und Richtung prinzipiell

6 Siehe z. B. auch Fuchs 2020, 37: „Es ist für mich, dass ich Schmerzen habe, wahrnehme, verstehe oder denke.“ 
frei bzw. spontan bestimmen kann (vgl. Cramer 1999, 22, 65 f.; Stekeler-Weithofer 2012, 112). Nur durch die so verstandene Aktivität des freiheitlich gelenkten Gedankenhervorbringens kann es seine Gedankengänge und Entscheidungen im Sinne genuin mentaler Verursachung mit intentionalem Bezug zum semantischen Gehalt von Zwecken, Gründen und (epistemischen) Normen - also anhand inhaltlicher und logischer Kriterien - entwickeln, in einem metastufigen Reflexionsprozess vorgegebene Gründe und Normen hinterfragen und somit „epistemische Verantwortung“ (Cramm 2008, 56) als Akteur im Raum der Gründe übernehmen.

An dieser Stelle lässt sich das schon angesprochene Problem verdeutlichen, mit dem sich die in den Neurowissenschaften vorherrschenden reduktiv-naturalistischen Auffassungen des Bewusstseins konfrontiert sehen. Sie können die hier skizzierten Eigenschaften des Denkens, die konstitutiv für alle Rationalität in lebensweltlichen und wissenschaftlichen Kontexten sind, nicht einholen und somit nur auf Kosten eines performativen Selbstwiderspruchs rationale Geltung in eigener Sache beanspruchen oder den nicht von der Hand zu weisenden Fortschritt der eigenen Disziplin in der Entwicklung von Neurotechnologien erklären. Denn weil im Rahmen reduktiv-naturalistischer Auffassungen jeder physische und jeder mentale Zustand (im Sinne seiner Reduzierbarkeit) durch eine hinreichende physische Ursache vollständig festgelegt ist und dabei die problematische Möglichkeit systematischer Überdetermination ${ }^{7}$ ausgeschlossen wird, sodass es neben dieser einen hinreichenden physischen Ursache für ein (physisches oder mentales) Ereignis keine zweite und damit auch keine mentale Ursache geben kann, bleibt kein Raum für genuin mentale Verursachung bzw. die kausale Relevanz mentaler Gehalte (z. B. Gründe). Gedankengänge wären durch die ,blinden“ Dispositionen des fundamentalen (neuronalen) Naturgeschehens vollständig festgelegt. Von dem für rationale Zusammenhänge unverzichtbaren Vermögen, Gedankengänge gemäß mentaler Verursachung anhand inhaltlicher Kriterien frei entwickeln zu können, könnte keine Rede mehr sein (vgl. Müller 2013, 137-139).

\subsection{Bewusstsein - Organismus - Natur: Die Bedingungsverhältnisse konkreter Subjektivität}

Dass Subjektivität in ihrer rückbezüglichen Bewusstseinsstruktur keine unmittelbaren Relationen zu etwas ihr Äußerem hat, heißt wiederum nicht, dass sie in einem solipsistischen Sinne in sich gefangen ist und überhaupt keinen Bezug zur

7 Siehe zu den Problemen systematischer Überdetermination Brüntrup 2012, $49 \mathrm{f}$. 
bewusstseinsunabhängigen Wirklichkeit hat. Zwar zeichnet sich das zur Rationalität befähigende Denkvermögen ja gerade durch eine starke Spontaneität aus, sodass ein denkendes Subjekt Inhalt und Richtung seiner Gedankengänge prinzipiell frei von Einflüssen bestimmen kann, die außerhalb des Wirklichkeitsmodus des Bewusstseins bzw. Denkens stehen. Jedoch - und hier liegt ein entscheidender Unterschied zwischen Denken und Erleben - steht es einem erlebenden Subjekt nicht frei, dass und was es erlebt. Auch wenn ein Subjekt, das Schmerzen und eine Rotwahrnehmung hat, diese Erlebensgehalte im Modus des Bewusstseins aktiv produziert, kann es in dieser konkreten Situation nicht willkürlich beeinflussen, dass es keine Schmerzen und keine oder eine andere Farbwahrnehmung hat (vgl. Cramer 1999, 28). Für die konkrete Inhaltlichkeit des Erlebens kann somit nicht die Bewusstseinsaktivität des Subjekts, sondern nur eine Instanz verantwortlich gemacht werden, die selbst kein Moment dieser Aktivität bzw. des Modus des Bewusstseins ist, aber dennoch mit diesem so verschränkt ist, dass sie die Erlebensinhalte bedingen kann. Diese für jedes erlebende Subjekt konstitutive Instanz ist sein Organismus. Der notwendige Bezug des Erlebens auf ein externes, seine Inhaltlichkeit bedingendes Moment bedeutet also, dass sich Subjektivität nicht in reiner Bewusstseinsaktivität erschöpft, sondern immer eingebettet in einen konkreten Organismus ist, mithin Subjektivität nur als konkrete, verkörperte Subjektivität in Form konkreter Lebewesen Pflanzen, Tiere, Menschen - auftritt (vgl. ders. 1999, 34-41; 2012, 103f.).

Da der Organismus dabei nicht nur als lebendiger Leib eines Lebewesens unauflöslich mit dessen Erleben verschränkt ist, sondern zugleich als Körper mit anderen raumzeitlichen Entitäten in Verbindung steht, stellt er die vermittelnde Schnittstelle zwischen Subjektivität in ihrer irreduziblen Bewusstseinsstruktur und der bewusstseinsunabhängigen Natur dar (vgl. ders. 1999, 43-45). Auch wenn sich ein Subjekt im rückbezüglichen Modus des Erlebens nicht direkt auf die subjektunabhängige Wirklichkeit beziehen kann, so nimmt es sie doch insofern mittelbar wahr, als es seine Erlebensgehalte immer abhängig von seiner leiblichen Verschränkung mit organismischen Strukturen produziert, die ihrerseits in direkter Wechselwirkung mit der subjektexternen Natur stehen (vgl. ders. 1999, 45-47; 2012, 116f.). ${ }^{8}$ Der nur menschlichen Subjekten zukommende Bewusst-

8 Dieses an Wolfgang Cramer orientierte Verständnis der äußeren Wahrnehmung ist durchaus kompatibel mit phänomenologischen Auffassungen, wie sie etwa bei Thomas Fuchs zu finden sind. Zwar stellt Fuchs die Beziehung des verkörperten Subjekts zur ihm begegnenden Umwelt ins Zentrum der Welterfahrung. Doch in einer gewissen Ähnlichkeit zu Cramer bestimmt er einerseits dem Subjekt zuzuschreibende „Wahrnehmungsvermögen“ und andererseits „Objekteigenschaften“ der subjektunabhängigen Natur als konstitutive Momente für die Entstehung konkreter Wahrnehmungsgehalte. Dabei wird das „Zusammenwirken“ dieser beiden Momente - ganz im 
seinsmodus des Denkens steht wiederum mit dem ihm vorausgesetzten Erleben in einem derartigen Bedingungsverhältnis, dass ein denkendes Subjekt seine Gedanken prinzipiell in Bezug auf Erlebtes, also auch äußerlich Perzipiertes, lenken und dieses dadurch begrifflich bestimmen kann (vgl. ders. 1999, 72). Dieses dreifache Bedingungsverhältnis (Natur - Organismus - Erleben - Denken) ermöglicht einen indirekten Bezug des Denkens auf die subjektunabhängige Wirklichkeit und ist somit auch konstitutive Voraussetzung für jede rationale Erkenntnis über diese.

Es ist also die Leiblichkeit bzw. die konstitutive Verschränkung mit organismischen Strukturen, die ein Subjekt so in der Welt verortet, dass es sie wahrnehmen, sich auf sie beziehen und auch mit ihr interagieren kann (vgl. Fuchs 2013, 31f., 40). Das heißt, dass der Organismus nicht nur - wie im Falle der äußeren Wahrnehmung - den indirekten Einfluss der subjektexternen Natur auf das Erleben vermittelt, sondern auch andersherum das Wirken des Bewusstseins auf die Natur über organismische Aktivität ermöglicht. Nur wenn ein Lebewesen abhängig von seinem Erleben, etwa einem Hungergefühl oder dem Wahrnehmen einer Gefahrenquelle, seinen Organismus spontan zu Aktivitäten bestimmen kann, kann es sich in eine für seine Selbsterhaltung erfolgreiche Interaktion mit der Welt bzw. dem ihm Begegnenden begeben, das durch das Wechselspiel von erlebensbedingtem Verhalten und verhaltensbedingtem Erleben $\mathrm{zu}$ seiner ihm spezifischen und bedeutsamen Umwelt konstituiert wird: Durch sein Verhalten verändert es seine Umwelt und bringt sich in immer neue Beziehungen zu ihr, sodass es immer neue Außenwahrnehmungen und Leibesempfindungen hat, die wiederum sein Verhalten beeinflussen (vgl. Cramer 1999, 47-50; 2012, 117 f.; Fuchs 2013, 113-116). Dabei ermöglicht die skizzierte Spontaneität des Denkens menschlichen Subjekten, aus ihrer ursprünglichen Eingebundenheit in dieses Wechselspiel heraustreten zu können. Durch das prinzipiell freie Lenken der Gedanken müssen sie ihr Tun nicht unmittelbar an Eigen- und Umweltwahrnehmungen ausrichten, sondern können die je konkrete Situation und die eigene Stellung in ihr bedenken, verschiedene Optionen abwägen und sich aufgrund von Zwecken, Werten und Gründen, die möglicherweise nichts mit der aktuellen Umwelterfahrung zu tun haben, zu Handlungen bestimmen (vgl. Cramer 1999, 74; Fuchs 2013, 119). Schließlich sei darauf hingewiesen, dass man in seinem Erleben, Denken, Verhalten und Handeln nicht nur in einer Beziehung zu seiner je eigenen Umwelt steht, sondern auch in intersubjektive Interaktionen mit anderen konkreten Subjekten tritt, wodurch sich ein gemeinsamer Sozialraum bzw. eine so-

Sinne Cramers - durch die Verkörperung des Subjekts, also durch seine Verschränkung mit organismischen Strukturen, ermöglicht bzw. vermittelt (vgl. Fuchs 2013, 46f.). 
ziale Mitwelt mit geteilten sozio-kulturellen Praktiken formt (vgl. Fuchs 2013, 187f., 265).

Die Möglichkeit psycho-physischer Wechselwirkungen, wie sie etwa im Erleben der Umwelt oder in der Aktualisierung eines Gedankens zu einer in der Welt wirksamen Handlung zum Tragen kommen, gründet auf einem Verhältnis von Bewusstsein und Organismus, dessen formale Bestimmung als notwendige Korrelation zu kurz greift. Eine solche konstatiert zwar zutreffend, dass das eine niemals ohne das andere sein kann, lässt aber dennoch - bei aller Notwendigkeit des korrelativen Auftretens - die unzureichende Möglichkeit eines Substanzdualismus offen, der Bewusstsein und Organismus nicht nur als aufeinander irreduzible, sondern auch als völlig getrennte Wirklichkeitszusammenhänge versteht und somit deren Wechselwirkung nicht plausibel fassen kann. ${ }^{9}$

Entscheidend ist vielmehr, dass Subjektivität in ihrem spezifischen Bewusstseinsmodus notwendig aus sich selbst heraus - nämlich durch die inhaltliche Bedingtheit des Erlebens - auf ihren Organismus als für sie konstitutives Moment verweist. Durch diese inhärente Differenz erweist sich verkörperte Subjektivität nicht als sekundäre Verbindung von Bewusstsein und Körper, sondern primär als binnendifferenzierte Einheit zweier zu unterscheidender (aber nicht getrennter), unaufhebbarer und aufeinander bezogener Momente. Sind Bewusstsein und Organismus nach diesem Verständnis zwei miteinander verschränkte Momente einer zugrundeliegenden Einheit, nämlich des konkreten Lebewesens, lässt sich ihre Wechselwirkung formal als gegenseitiges Bedingungsverhältnis bestimmen, das die Differenz in der Einheit gewissermaßen konstituiert. So tritt Bewusstsein immer nur als ein Moment im Rahmen der psycho-physischen Einheit eines Lebewesens auf und ist, wie gezeigt, konstitutiv durch das physische Moment, den Organismus, bedingt. Dieser wiederum ist als Moment eines Lebewesens nicht nur physisch bzw. nicht nur ,bloße‘ Natur. Sondern er ist dadurch zu einem lebendigen Leib spezifizierte Natur, dass er einerseits das Bewusstseins-Moment des Lebewesens bedingt und andererseits selbst durch dieses bedingt wird, also organismische Prozesse nicht nur durch physische, sondern auch durch psychische Faktoren, z. B. die Herzfrequenz von erlebtem Stress, mitbestimmt werden. ${ }^{10}$

9 Siehe zum Problem der psycho-physischen Wechselwirkung in substanzdualistischen Positionen Brüntrup 2012, 44-64.

10 Vgl. ausführlich zur Bestimmung des konkreten Subjekts bzw. Lebewesens als binnendifferenzierte Einheit zweier sich bedingender Momente Cramer 1999, 41-45. 


\subsection{Die Verschränkung psychischer und physischer Aspekte in gesamtorganismischen Lebensvollzügen}

Für die anschließenden Überlegungen zu Neurotechnologien ist es von Interesse, diese formalen Verhältnisbestimmungen zu konkretisieren und dabei das Phänomen psycho-physischer Wechselwirkung jenseits substanzdualistischer Klüfte adäquat zu fassen. Mentales und Physisches stehen sich nicht als konkurrierende Kausalfaktoren zweier getrennter Bereiche gegenüber, sondern sind in gesamtorganismischen Lebensprozessen eines Lebewesens verschränkte Aspekte und werden durch diese gewissermaßen zu einer differenzierten Einheit integriert, wobei sie mit ihren kausalen Dispositionen die sie integrierenden Lebensprozesse beeinflussen. Dass solche gesamtorganismischen Lebensvollzüge folglich nicht allein durch physische Faktoren bedingt sind und daher nicht rein wirkkausal beschrieben werden können, sieht man schon daran, dass physische Prozesse, die in einen lebendigen Organismus eingebettet sind, anders ablaufen als in der ,bloßen' außerorganismischen Natur. ${ }^{11}$

Vielmehr weisen solche gesamtorganismischen Lebensvollzüge, mit denen ein verkörpertes Subjekt im Spannungsfeld psychischer und physischer Bedürfnisse in eine dynamische Interaktion mit seiner Umwelt tritt, spezifische Kausalstrukturen auf, die sich mit Thomas Fuchs’ Konzept zirkulärer Kausalität (vgl. Fuchs 2013, 121-132) näher bestimmen lassen.

Gemäß der vertikalen zirkulären Kausalität lassen sich in einem Lebewesen vom Organismus als Ganzen, über physiologische Systeme und Organe bis hin zu Zellen, Molekülen und Atomen - verschiedene Ebenen unterscheiden, die in einer vertikalen Hierarchie zueinander stehen und sich wechselseitig beeinflussen. Durch vertikale Kausalschleifen wirkt jede Ebene bottom-up auf die nächst höhere Ebene und über diese immer weiter bis auf den Gesamtorganismus in seinen Lebensvollzügen, während gleichzeitig dieser und jede andere Ebene auch topdown auf die niedrigeren Ebenen wirken (vgl. ebd., 122f.). Dabei genießt jede Ebene eine gewisse Autonomie und trägt zum übergeordneten Ganzen bei (z. B. zelluläre Prozesse zur Organfunktion), während dieses umgekehrt die Funktion seiner Komponenten beeinflusst und erst ermöglicht (z. B. das Organ die Prozesse seiner Zellen) (vgl. ebd., 112, 122). Der top-down-Richtung der Kausalschleifen ist vorrangig eine formierende Wirkung auf die untergeordneten Teile zuzusprechen

11 Während beispielsweise Eisen normalerweise irreversibel oxidiert, ist es durch seine Hämoglobin-Bindung im Rahmen organismischer Strukturen dazu fähig, Sauerstoff reversibel zu binden. 
und genau hier ist auch die Wirksamkeit mentaler Aspekte, sofern man diese überhaupt isoliert betrachten kann, anzusiedeln (vgl. ebd., 123f.).

Durch diese kausalen Verschränkungen lassen sich Veränderungen des organismischen Gesamtzustandes durch verschiedene Manipulationen herbeiführen, die sich sowohl hinsichtlich des Moments (mental oder physisch) als auch hinsichtlich der vertikalen Ebene unterscheiden können, an dem bzw. auf der sie ansetzten. So lässt sich etwa ein Angstzustand, gewissermaßen ein Aspekt des gesamtorganismischen Zustands, Psychopharmaka mildern, deren unmittelbare Wirkung auf den Neurotransmitterhaushalt subkortikaler Hirnstrukturen sekundär zu Veränderungen höherstufiger neuronaler Prozesse führt (bottom-up) und dabei im Sinne der psycho-physischen Verschränkung das emotionale Erleben beeinflusst. Andersherum setzt ein beruhigendes Gespräch primär am mentalen Moment an, mildert über eine veränderte kognitive Einschätzung top-down den Angstzustand und führt gleichzeitig über die psycho-physische Verschränkung zu physiologischen Veränderungen, die sich ebenfalls top-down von kortikalen über subkortikale bis hin zu vegetativen Abläufen fortsetzten (vgl. ebd., 125, 285-287).

Auch die zweite Form zirkulärer Kausalität, nämlich die horizontale zirkuläre Kausalität, unterscheidet sich grundlegend von linearen Ursache-Wirkungs-Zusammenhängen, die in naturwissenschaftlichen Erklärungen und naturalistischen Deutungen eine zentrale Rolle einnehmen. Zum einen zeigt sie sich in den zirkulären Wechselwirkungen, die auf den verschiedenen organismischen Ebenen, etwa in Form hormoneller Rückkopplungsschleifen, stattfinden. Zum anderen bezeichnet sie die Dynamik, in der diese Ebenen mit der Umwelt wechselwirken, sei es basal den Stoffwechsel oder sei es Interaktionen des gesamten Lebewesens mit seiner Umwelt betreffend (vgl. ebd., 125f.). Gerade Letzteres zeigt in seinem oben genannten Wechselspiel aus erlebensbedingtem Verhalten und verhaltensbedingtem Erleben sowohl die Verschränkung psychischer und physischer Aspekte in Lebewesen und ihren Vollzügen als auch die Nicht-Linearität organismischer Kausalzusammenhänge.

Integrale zirkuläre Kausalität bezeichnet den Zusammenhang, dass in einem verkörperten Subjekt sowohl horizontale als auch vertikale Kausalschleifen mit all ihren physischen und psychischen Aspekten zu teils angeborenen, teils erlernten Vermögen integriert sind, die in Anlehnung an Aristoteles als gesamtorganismische Fähigkeiten verstanden und vom verkörperten Subjekt aktiv aktualisiert werden können. So werden etwa in Handlungs- und Verhaltenskontexten neuronale Strukturen, zelluläre Transmissionen, Muskeln, Sehnen usw. in vertikaler Kausalität zu einer funktionalen Einheit gebündelt, die das Lebewesen für eine bestimmte horizontale Interaktion mit der Umwelt aktivieren kann (vgl. ebd. 126-128). Freilich müssen beim Erlernen und Vollzug von Vermögen auch Aspekte integriert werden, die vorrangig dem mentalen Moment des Lebewesens 
zuzuordnen sind: etwa auditive, visuelle und propriozeptive (d.h. die räumliche Stellung des Leibes betreffende) Erlebensgehalte beim Erlernen und Spielen eines Instruments.

Wie sich in diesen gesamtorganismischen Lebensvollzügen die Funktion des Gehirns, dem zentralen Ansatzpunkt von Neurotechnologien, charakterisieren lässt, kann hier nur angedeutet werden. ${ }^{12}$ Insofern sich Bewusstsein nicht vollständig auf neuronale bzw. organismische Strukturen zurückführen lässt, kann das Gehirn nicht in einem neurokonstruktivistischen Sinne als Produzent des Bewusstseins begriffen werden. Vielmehr ist es als Organ eines psycho-physischen Lebewesens immer schon eingebettet in den unhintergehbaren Zusammenhang von Subjektivität, Organismus und Umwelt. Dabei stellt es mit seinen komplexen neuronalen Verschaltungen und zahlreichen (z. B. neuroendokrinen) Rückkopplungsschleifen zu verschiedensten organismischen Ebenen die notwendigen Strukturen zur Verfügung, damit etwa Einflüsse verschiedener Kausalebenen und -schleifen wechselseitig übertragen oder diese zu größeren integralen Einheiten als Grundlagen bestimmter Vermögen gebündelt werden können. Kurz gesagt fungiert das Gehirn in den zirkulär-kausalen Lebensprozessen als zentrales „Vermittlungsorgan“ (z. B. Fuchs 2013, 131; 2020, 195), in dem „alle Kreisprozesse zusammenlaufen und verknüpft werden“ (ders. 2013, 152).

Nehmen wir zum Abschluss dieser Skizze konkreter Subjektivität vor dem Hintergrund der gerade erfolgten kausaltheoretischen Konkretisierungen der Bedingungsverhältnisse von Bewusstsein, Organismus und Natur noch einmal die formale Bestimmung des Bewusstseins als genuin aus und für sich produzierende Aktivität eines Subjekts in den Blick. Sie erweist sich nun als Aktivität eines verkörperten Subjekts, das immer schon in dynamische Lebensprozesse und Interaktionen mit seiner Umwelt eingebettet ist (vgl. ebd., 153). Anders gesagt ist das Zeugen von Bewusstseinsgehalten integraler Bestandteil gesamtorganismischer zirkulär-kausaler Lebensvollzüge und dabei durch andere mentale Momente (z. B. Gedanken) und physische Faktoren (z. B. neuronale Strukturen) bedingt. Die auf diese Weise produzierten Bewusstseinsgehalte gehen dann ihrerseits als kausal relevante Aspekte in die gesamtorganismischen Lebensprozesse ein, wobei sie über zirkulär-kausale Zusammenhänge auf andere mentale und physische Aspekte wirken. Am Beispiel des Klavierspielens lässt sich die kausale Relevanz mentaler Gehalte im Sinne genuin mentaler Verursachung dann so denken, dass ein Pianist im gesamtorganismischen Vollzug des Spielens sein Bewusstsein aktiv auf den Notentext lenkt und abhängig von den je wahrgenommenen Notenzeichen über integral-kausale Verknüpfungen die entspre-

12 Siehe für eine ausführliche Darstellung Fuchs 2013, 133-184. 
chenden Handlungsvermögen aktiviert. Zudem passt er diese an seine im Vollzug erzeugten Höreindrücke - neue kausal relevante Bewusstseinsgehalte - an.

Auch der Bewusstseinsmodus des Denkens lässt sich nur als Aktivität verstehen, die in gesamtorganismische Vollzüge integriert ist (vgl. ebd., 65f., 107, 227). Dass er dabei von anderen Momenten dieser Vollzüge beeinflusst werden kann, zeigt sich, wenn man beispielsweise abhängig von seinem Hungergefühl über die nächste Mahlzeit nachdenkt. Gleichzeitig ermöglicht Denken als Aktivität des freiheitlich gelenkten Gedankenhervorbringens, wie oben schon angedeutet, dass ein verkörpertes Subjekt aus seiner Einbettung in den je konkreten Lebensvollzug gewissermaßen heraustreten und sich von diesem distanzieren, mithin trotz Hungergefühl über den Satz des Pythagoras nachdenken kann (vgl. Cramer 1999, 74).

Diese Fähigkeit zur Distanzierung vom je konkreten Lebensvollzug verbürgt zugleich die lebensweltliche Freiheitserfahrung, nicht - wie von reduktiv-naturalistischen Auffassungen nahegelegt - vollständig durch wirkkausale Faktoren des (neuronalen) Naturgeschehens determiniert zu sein, sondern sich an Gründen, Zwecken und Werten orientierend in einem libertarischen Sinne so frei entscheiden zu können, dass man eine echte zukunftsoffene Wahlmöglichkeit zwischen nicht festgelegten Handlungsoptionen hat, also in einer gegebenen Situation so oder anders handeln kann (vgl. Müller 2018, 90 -93). Freilich ist ein verkörpertes Subjekt in seinem Tun und Entscheiden motiviert durch zahlreiche Faktoren seines konkreten Lebensvollzugs, etwa durch Leibesempfindungen, Triebe, Neigungen, Emotionen, die individuelle Lerngeschichte oder gesellschaftliche Konventionen. Doch das Vermögen, Inhalt und Richtung seiner Gedanken prinzipiell frei bestimmen zu können, ermöglicht es, sich in ein kritisch reflektierendes Verhältnis zu solchen Motiven setzten und sich von ihnen distanzieren zu können, sie also nicht unmittelbar handlungswirksam werden lassen zu müssen, sondern sie verwerfen oder sich ihnen bewusst überlassen, oder seinem Tun und Entscheiden gar andere, nicht unmittelbar durch den konkreten Lebensvollzug bedingte Gründe und Zwecke geben zu können (vgl. Cramer 1999, 77-80; Keil 2018, 134f., 139f.).

\section{Neurotechnologien im Kontext konkreter Subjektivität}

Dadurch dass die skizzierte Theorie konkreter Subjektivität einerseits Bewusstsein in seiner irreduziblen Grundstruktur und andererseits seine konstitutive Einbettung in organismische Strukturen und damit in einen technisch manipulierbaren 
Naturzusammenhang berücksichtigt, erweist sie sich als eine anthropologisch adäquate Perspektive, um den Einfluss neurotechnologischer Anwendungen auf den Menschen in seiner psycho-physischen Verfasstheit und somit auch auf spezifische und irreduzible Eigenschaften von Subjektivität in den Blick zu nehmen.

Es wurde dafür argumentiert, dass sich der spezifische Zusammenhang des Bewusstseins nicht vollständig auf neuronale bzw. wirkkausal-funktionale Prozesse reduzieren lässt. Demnach ist auch bei noch so großem neurowissenschaftlichem und technischem Fortschritt eine direkte und vollständig kontrollierende Einflussnahme auf geistig-psychische Aspekte menschlicher Lebensvollzüge (kognitive Fähigkeiten, Emotionen etc.) prinzipiell nicht möglich. Anders gesagt können alle Forschungsziele und Vorstellungen, die eine vollständige Reduktion des Bewusstseins auf kausal-funktionale Strukturen voraussetzten, - etwa eingangs angedeutete Visionen eines wechselseitigen Transfers von Bewusstseinsinhalten zwischen Gehirn und Chips bzw. Festplatten - mit dem Verweis auf ihre prinzipiell unmögliche Realisierbarkeit zurückgewiesen werden. ${ }^{13}$

Der Einfluss einer neurotechnologischen Anwendung auf geistig-psychische Aspekte kann also nicht in einem direkten, linear-kausalen Zusammenhang gedacht werden, sondern muss vielmehr über das Bedingungsverhältnis von Organismus und Bewusstsein bzw. ihre dynamische Verschränkung in zirkulärkausalen Lebensprozessen verstanden werden. ${ }^{14}$ Da nun gerade das Gehirn in besonderem Maße relevant für spezifische Bewusstseinsaspekte (kognitive Fähigkeiten, Wahrnehmungen, Emotionen etc.) ist, lassen sich diese freilich durch Neurotechnologien beeinflussen. Weil solche Bewusstseinsaspekte aber immer in gesamtorganismische Lebensvollzüge eingebettet sind, bei denen zahlreiche neuronale, aber auch andere organismische Strukturen, andere psychische Aspekte und Umwelteinflüsse über zirkuläre Kausalschleifen beteiligt sind, moduliert die technische Manipulation einer neuronalen Struktur nur einen von vielen, sich dynamisch bedingenden Faktoren, sodass eine gezielte und steuerbare Einflussnahme etwa auf kognitive Fähigkeiten oder Wahrnehmungen zumindest nicht unproblematisch ist.

13 Ein solcher Bewusstseinstransfer würde im allgemeinen reduktiv-naturalistischen Rahmen entweder eine Form der Identitätstheorie oder des metaphysischen Funktionalismus voraussetzen, die sich beide mit unüberwindbaren Schwierigkeiten konfrontiert sehen (vgl. ausführlich Tobias Müllers Beitrag im vorliegenden Band).

14 Man denke an das oben genannte Beispiel der anxiolytischen Pharmakotherapie, bei der eine direkte Modulation physiologischer Abläufe indirekt auf das mit ihnen verschränkte affektive Erleben wirkt. 
Es verwundert daher nicht, dass Neurotechnologien momentan vor allem im Kontext eines medizinischen Störungsbeseitigungs- oder Störungsvermeidungswissens eingesetzt werden: Wenn es im Falle einer spezifischen Störung des Lebensvollzugs gelingt, diese auf eine möglichst isolierte Fehlfunktion einer physiologischen Struktur zurückzuführen, die sich somit als eine notwendige Bedingung für den gestörten Aspekt des Lebensvollzugs erweist, kann durch medizinisch-technische Mittel versucht werden, die Fehlfunktion auf physiologischer Ebene und damit die Störung des Lebensvollzugs zu beheben (vgl. Janich 2013, $94 \mathrm{f}$.).

Genau so lässt sich beispielsweise der erfolgreiche Einsatz von Cochlea-Implantaten verstehen. Wenn der Hörverlust, eine spezifische Störung des Lebensvollzugs, allein durch den isolierten Ausfall des Innenohrs bedingt ist, kann ein Cochlea-Implantat diesen Ausfall zumindest teilweise kompensieren und zum Wiedererlangen des Gehörs führen, indem es akustische Signale registriert, verarbeitet und abhängig davon den Hörnerv stimuliert. Dabei sollte dieser Zusammenhang nicht im reduktiv-naturalistischen Sinne gedeutet werden, also dass im Menschen als rein kausal-funktionales Gefüge ein gestörter Teil ausgetauscht wurde und nun am Ende einer wiederhergestellten Kausalkette erneut eine Hörwahrnehmung verursacht wird, die nicht mehr als das Epiphänomen eines neuronalen Wirkverlaufs ist. Vielmehr bedeutet vor dem Hintergrund konkreter Subjektivität der Hörverlust zunächst die Unfähigkeit, ein spezifisches Vermögen auszuüben, das in die zirkulär-kausale Interaktion eines verkörperten Subjekts mit seiner Umwelt eingebunden ist. Durch den Innenohrschaden wird eine für das Hörvermögen notwendige horizontale Kausalschleife an dem spezifischen Punkt unterbrochen, an dem der Naturzusammenhang in Form von Schallwellen auf den Organismus wirkt. Durch das Cochlea-Implantat wird die Lücke in der Kausalschleife gewissermaßen geschlossen, sodass das Subjekt wieder über das Vermögen verfügt, abhängig von auf seinen Organismus einwirkenden Schallwellen Hörwahrnehmungen aktiv zu erzeugen. Obwohl das Implantat Schallwellenmuster nicht ansatzweise so differenziert in eine Erregung des Hörnervs umwandeln kann wie ein funktionierendes Innenohr, lernt das Subjekt, auch abhängig von diesen gröberen Einflüssen auf seinen Organismus für sich so klare Höreindrücke zu produzieren, dass sie zum Verständnis gesprochener Sprache ausreichen (vgl. z. B. Clausen 2017, 153).

Ein weiteres Erfolgsbeispiel neurotechnologischer Anwendungen ist die Tiefe Hirnstimulation beim Morbus Parkinson. Bei dieser Erkrankung kann es aufgrund multifokaler Degenerationen des Gehirns, entsprechend seiner zentralen Rolle im menschlichen Lebensvollzug, zu verschiedenen Störungen desselben, nämlich von psychischen Erkrankungen und kognitiven Einschränkungen über motorische und vegetativ-autonome Störungen bis hin zu Schlaf-, Geruchs- und Ge- 
schmackstörungen kommen (vgl. z. B. Berg 2019, 590). In diesem weiten Spektrum an Symptomen ist es gelungen, die motorischen Beschwerden, die sich u.a. in einem starken Zittern und einer Verlangsamung willkürlicher Bewegungen äußern, mit einer isolierten Störung einer Funktionsschleife in einem Kerngebiet des Gehirns, den Basalganglien, in Verbindung zu bringen. Durch eine elektrische Stimulation an entsprechender Stelle wird die unterbrochene Kausalschleife zumindest so geschlossen, dass der Patient wieder flüssigere Bewegungen ausführen kann. Bei dieser Anwendung wird der Zusammenhang des Störungsbeseitigungswissens insofern besonders deutlich, als man die genaue Wirkung der elektrischen Stimulation auf die entsprechende Hirnstruktur nicht nachvollziehen kann bzw. nicht kennt (vgl. z. B. Christen 2017, 123; Gharabaghi/Freudenstein/ Tatagiba 2005, 64), jedoch durch die Beseitigung der Störung des Lebensvollzugs weiß, dass sie wirkt.

Der Rolle des Gehirns, zwischen verschiedenen an unterschiedlichen Aspekten des Lebensvollzugs beteiligten Kausalschleifen zu vermitteln, entspricht die komplexe Vernetzung seiner Strukturen. So weist auch die beim Morbus Parkinson stimulierte Funktionsschleife zahlreiche Verbindungen $\mathrm{zu}$ anderen Hirnstrukturen auf (vgl. z. B. Gharabaghi/Freudenstein/Tatagiba 2005, 60-63) und es sollte weder verwundern, dass es durch die Hirnstimulation zu unerwünschten Auswirkungen auf verschiedene, auch geistig-psychische Aspekte des Lebensvollzugs (z. B. kognitive Fähigkeiten, affektives und emotionales Erleben) kommen kann, noch dass diese Auswirkungen individuell stark, etwa von depressiven bis hin zu manischen Stimmungen, variieren (vgl. Davis et al. 2017). Denn gerade Aspekte des affektiven, emotionalen und leiblichen Erlebens sind im dynamischen Gesamtzusammenhang eines in der Welt agierenden Lebewesens durch so viele physische und psychische Faktoren bedingt, dass man Veränderungen dieser Erlebensaspekte im Rahmen Tiefer weder sicher vorhersehen noch im Nachhinein eindeutig auf diese zurückführen kann. So sollte bei der Beurteilung von Einzelfällen auch Vorsicht walten, wenn es um die Charakterisierung einer Stimmungs- oder Verhaltensänderung als klare Nebenwirkung einer Stimulationstherapie geht. Führt etwa ein Patient mit einem Hirnstimulator ein in bisher unbekannter Weise aktives Nachtleben mit Strip-Club-Besuchen (vgl. Christen/Müller 2015, 161, Case 3), könnte das zwar an einer durch die Stimulation bedingten Veränderung seiner sexuellen Appetenz liegen - allgemein formuliert führte in diesem Fall die technische Modulation zu einem veränderten leiblichen Erleben, dem das Subjekt mit dem Ausüben einer durch seine konkrete soziokulturelle Einbettung nahegelegten Praktik begegnet. Aber genauso gut könnte diese Verhaltensänderung durch eine Verbesserung einer latent depressiven Stimmung motiviert sein. Und darüber, ob diese Verbesserung wiederum hauptsächlich durch die Tiefe Hirnstimulation oder durch bestimmte lebensweltliche 
Erfahrungen des Patienten bedingt ist, sollte nicht vorschnell geurteilt werden. Von einer gar ,erzwungenen` Verhaltensänderung durch Hirnstimulationen kann, wenn überhaupt, erst dann die Rede sein, wenn nachweislich das Reflexionsvermögen als Grundlage des skizzierten Freiheitsvermögens gestört ist, also man sich etwaige durch die Therapie beeinflusste Veränderungen des Erlebens (z. B. eine gesteigerte sexuelle Appetenz) nicht mehr reflektierend $\mathrm{zu}$ Bewusstsein bringen und sich somit nicht mehr von Handlungsimpulsen, die durch diese Veränderungen bedingt sind, distanzieren kann. Diese kurzen Überlegungen sollten verdeutlichen, inwiefern sich der Ansatz konkreter Subjektivität auch als Reflexionsrahmen für konkrete Fälle eignet, um etwa Verhaltens- oder Stimmungsänderungen eines Patienten im Rahmen einer neurotechnologischen Anwendung differenziert beurteilen zu können.

Zuletzt seien noch die Versuche genannt, gelähmten Menschen zu ermöglichen, durch in das Gehirn implantierte Elektroden externe Prothesen (z. B. eine Roboterhand) anzusteuern (vgl. Duncan 2005). Liegt die organische Ursache der Lähmung nicht in der primär-motorischen Hirnrinde, ist also die Kausalschleife willkürlicher Bewegungen distaler, etwa auf Höhe des Rückenmarks, unterbrochen, weist diese Hirnregion nach wie vor spezifische Erregungsmuster auf, wenn der gelähmte Mensch versucht, eine Bewegung zu initiieren. Durch implantierte Elektroden, so das Ziel, können die mit Handlungsintentionen einhergehenden Erregungsmuster decodiert, an einen Computer weitergegeben, verarbeitet und letztlich zur Steuerung einer externen Prothese genutzt werden. Der Ansatz konkreter Subjektivität eröffnet die Möglichkeit, die ersten erzielten Erfolge so zu deuten, dass es der Patient mittels seiner Handlungsintentionen (im Sinne genuin mentaler Verursachung) schafft, die Prothese zu steuern. Handlungsintentionen sind demnach als kausal wirksame mentale Zustände mit physischen Aspekten in den Kausalschleifen willkürlicher Bewegungen verschränkt, und im Falle des primär-motorischen Kortex gelingt eine einigermaßen konsistente korrelative Zuordnung zwischen physischen Erregungsmustern und mentalen Intentionen, sodass er sich als Ansatzpunkt für besagte decodierende Elektroden eignet. Auch wenn die Erfolge solcher Anwendungen sicher nicht als empirischer Beweis für die Realität mentaler Verursachung gelten dürfen, wäre eine naturalistische Deutung, die Intentionen jegliche kausale Relevanz abspricht, zumindest problematischer als die hier vorgestellte (vgl. dazu auch Falkenburg 2013, 60).

\section{Resümee}

Der naturwissenschaftliche Zugang zur Ergründung des Menschen basiert auf einer spezifischen Perspektive, die allein aus methodischen Gründen nur wirk- 
kausal-funktionale Zusammenhänge thematisieren kann und daher auf anatomisch-physiologische Strukturen fokussiert. Zweifelsohne ist diese Perspektive die Basis für den Erfolg der modernen Medizin, bestimmte dieser kausal-funktionalen Zusammenhänge durch technisch-medizinische Mittel gezielt modulieren und so Krankheiten therapieren zu können (vgl. Maio 2008, 216f.). Dass die naturwissenschaftliche Perspektive dabei sowohl geistig-psychische Aspekte des Menschen als auch deren leibliche Verschränkung mit organismischen Strukturen ausblendet, mag in vielen medizinischen Kontexten eine untergeordnete Rolle spielen. Doch bei der neurowissenschaftlichen Erforschung des Gehirns und dem damit einhergehenden medizinisch-technischen Zugriff auf neuronale Strukturen stellt sich die Frage nach dem Status des menschlichen Bewusstseins mit besonderer Dringlichkeit.

Dabei rechtfertigt der Erfolg der naturwissenschaftlich-technischen Beherrschung des Menschen und seines Gehirns keineswegs, sein psychisches Moment auf kausal-funktionale, neuronale Abläufe zu reduzieren bzw. das Bewusstsein vollständig zu naturalisieren. Im Gegenteil: Es ist gerade der sich jeder Reduktion entziehende Zusammenhang des menschlichen Denkvermögens, der im frei gelenkten Gedankenhervorbringen eine rationale Wissenschaftspraxis und damit einen erfolgreichen technischen Zugriff auf den Menschen überhaupt erst ermöglicht.

Möchte man solche Zugriffe aus anthropologischer Perspektive begleiten, was gerade bei technischen Anwendungen am Gehirn, dem in besonderem Maße bewusstseinsrelevanten Organ, unverzichtbar ist, darf der Abstraktionsgrad der naturwissenschaftlichen Perspektive nicht übersehen und diese nicht $\mathrm{zu}$ einem reduktiv-naturalistischen Menschenbild verabsolutiert werden. Vielmehr muss der Mensch in seiner psycho-physischen Verfasstheit mitsamt den irreduziblen Eigenschaften seines Bewusstseins betrachtet werden. Wie das gelingen kann, haben die Überlegungen zur konkreten Subjektivität gezeigt.

Begreift man den Menschen als verkörpertes Subjekt, also als binnendifferenzierte Einheit, in der Bewusstsein und Organismus als zwei einander bedingende Momente verschränkt sind, eröffnet sich die Möglichkeit, den Einfluss neurotechnologischer Anwendungen auf den ganzen Menschen, seine physischen und psychischen Aspekte in den Blick zu nehmen, ohne letztere durch eine naturalistische Reduktion ihrer spezifischen Charakteristika zu berauben. Dabei bietet diese anthropologische Perspektive Raum, um bei der Beurteilung heutiger und künftiger neurotechnologischer Interventionen sowohl neue empirische Erkenntnisse über den Menschen und sein Gehirn als auch weiterführende leib-, bewusstseins- und subjektphilosophische Überlegungen berücksichtigen $\mathrm{zu}$ können. 


\section{Literatur}

Berg, Daniela ( ${ }^{4}$ 2019): Krankheiten der Basalganglien, in: Hacke, Werner (Hg.): Neurologie, Berlin/Heidelberg, 589-623.

Berger, Theodore et al. (2005): Restoring lost cognitive function. Hippocampal-cortical neural prostheses, in: IEEE Engineering in Medicine and Biology Magazine 24/5, 30-44.

Brüntrup, Godehard ( $\left.{ }^{4} 2012\right)$ : Das Leib-Seele-Problem. Eine Einführung, Stuttgart.

Christen, Markus (2017): Klinische und ethische Fragen der Neuromodulation, in: Erbguth, Frank/Jox, Ralf (Hg.): Angewandte Ethik in der Neuromedizin, Berlin, 117-128.

Christen, Markus/Müller, Sabine (2015): Effects of brain lesions on moral agency. Ethical dilemmas in investigating moral behavior, in: Current Topics in Behavioral Neurosciences 19, $159-188$.

Clausen, Jens (2008): Gehirn-Computer-Schnittstellen. Anthropologisch-ethische Aspekte moderner Neurotechnologien, in: Ders./Müller, Oliver/Maio, Giovanni (Hg.): Die ,Natur des Menschen' in Neurowissenschaft und Neuroethik, Würzburg, 39-58.

Clausen, Jens (2017): Neuroprothesen und Gehirn-Computer-Schnittstellen, in: Erbguth, Frank/Jox, Ralf (Hg.): Angewandte Ethik in der Neuromedizin, Berlin, 151-161.

Clausen, Jens/Müller, Oliver/Maio, Giovanni (2008): Vorwort, in: Dies. (Hg.): Die ,Natur des Menschen' in Neurowissenschaft und Neuroethik, Würzburg, $7 \mathrm{f}$.

Cramer, Wolfgang ( $\left.{ }^{4} 1999\right)$ : Grundlegung einer Theorie des Geistes, Frankfurt a. M.

Cramer, Wolfgang (2012): Die absolute Reflexion. Schriften aus dem Nachlass, Frankfurt a. M.

Cramm, Wolf-Jürgen (2008): Zur kategorialen Differenz von Vernunft und Natur, in: Ders./Keil, Geert (Hg.): Der Ort der Vernunft in einer natürlichen Welt. Logische und anthropologische Ortsbestimmungen, Weilerswist, 44-57.

Davis, Rachel et al. (2017): Disambiguating the psychiatric sequelae of parkinson's disease, deep brain stimulation, and life events. Case report and literature review, in: American Journal of Psychiatry 174/1, 11-15.

Duncan, David (2005): Hirnimplantate. Fernsteuerung durch Gedanken, in: Technology Review $3,72-78$.

Elger, Christian et al. (2004): Das Manifest. Elf führende Neurowissenschaftler über Gegenwart und Zukunft der Hirnforschung, in: Gehirn und Geist 6, 30-37.

Falkenburg, Brigitte ( $\left.{ }^{4} 2013\right)$ : Was heißt es, determiniert zu sein? Grenzen der naturwissenschaftlichen Erkärung, in: Sturma, Dieter (Hg.): Philosophie und Neurowissenschaften, Frankfurt a.M., 43-74.

Fuchs, Thomas ( $\left.{ }^{4} 2013\right)$ : Das Gehirn - ein Beziehungsorgan. Eine phänomenologisch-ökologische Konzeption, Stuttgart.

Fuchs, Thomas (2020): Verteidigung des Menschen. Grundfragen einer verkörperten Anthropologie, Berlin.

Gazzaniga, Michael (2005): The ethical brain, New York.

Gharabaghi, Alireza/Freudenstein, Dirk/Tatagiba, Marcos (2005): Wiederherstellung der Funktion. Modulation von Hirnfunktionen durch Neuroprothesen, in: Engels, Eve-Marie/Hildt, Elisabeth (Hg.): Neurowissenschaften und Menschenbild, Paderborn, 57-75.

Hansmann, Otto (2018): Begriff und Geschichte des Transhumanismus, in: Göcke, Benedikt/Meier-Hamidi, Frank (Hg.): Designobjekt Mensch. Die Agenda des Transhumanismus auf dem Prüfstand, Freiburg, 25-51. 
Hildt, Elisabeth (2005): Computer, Körper und Gehirn. Ethische Aspekte eines Wechselspiels, in: Engels, Eve-Marie/Dies. (Hg.): Neurowissenschaften und Menschenbild, Paderborn, $121-137$.

Hoffmann, Thomas (2013): Hermeneutischer Naturalismus, in: Gerhard, Myriam/Zunke, Christine (Hg.): Die Natur denken, Würzburg, 27-55.

Holtzheimer, Paul/Mayberg, Helen (2011): Deep brain stimulation for psychiatric disorders, in: Annual Review of Neuroscience 34/1, 289-307.

Janich, Peter ( $\left.{ }^{4} 2013\right)$ : Der Streit der Welt- und Menschenbilder in der Hirnforschung, in: Sturma, Dieter (Hg.): Philosophie und Neurowissenschaften, Frankfurt a. M., 75-96.

Keil, Geert (2018): Willensfreiheit und Determinismus, Stuttgart.

Maio, Giovanni (2008): Medizin und Menschenbild. Eine Kritik anthropologischer Leitbilder der modernen Medizin, in: Ders./Clausen, Jens/Müller, Oliver (Hg.): Mensch ohne Maß? Reichweite und Grenzen anthropologischer Argumente in der biomedizinischen Ethik, Freiburg/München, $215-229$.

Mi, Kuanqing (2016): Use of deep brain stimulation for major affective disorders (Review), in: Experimental and Therapeutic Medicine 12/4, 2371-2376.

Mitscherlich-Schönherr, Olivia: Ethisch-anthropologische Weichenstellungen bei der Entwicklung von tiefer Hirnstimulation mit ,Closed Loop', im vorliegenden Band.

Müller, Oliver/Rotter, Stefan (2017): Neurotechnology. Current developments and ethical issues, in: Frontiers in Systems Neuroscience 11/93, 1-5.

Müller, Tobias (2013): Zu Möglichkeit und Wirklichkeit mentaler Verursachung, in: Philosophisches Jahrbuch 120/1, 131-143.

Müller, Tobias (2015): Naturwissenschaftliche Perspektive und menschliches Selbstverständnis. Eine wissenschaftsphilosophische Analyse zur Unverzichtbarkeit lebensweltlicher Qualitäten, in: Ders./Schmidt, Thomas (Hg.): Abschied von der Lebenswelt? Zur Reichweite naturwissenschaftlicher Erklärungsansätze, Freiburg/München, 31-52.

Müller, Tobias (2018): Der moderne Naturbegriff, unser lebensweltliches Freiheitsverständnis und die Grenzen der Freiheit, in: Jahrbuch Praktische Philosophie in globaler Perspektive 2, 89-110.

Müller, Tobias: Die transhumanistische Utopie des Mind-Uploading und die Grenzen der technischen Manipulation menschlicher Subjektivität, im vorliegenden Band.

Nagel, Thomas (1974): What is it like to be a bat?, in: The philosophical review 83/4, $435-450$.

Stekeler-Weithofer, Pirmin (2012): Denken. Wege und Abwege in der Philosophie des Geistes, Tübingen. 
